\title{
Multiplicity of monetary steady states
}

\author{
Ryoji Hiraguchi \\ Chiba University and The Canon Institute for Global Studies \\ Keiichiro Kobayashi \\ Keio University and The Canon Institute for Global Studies
}

September 27, 2014

※Opinions expressed or implied in the CIGS Working Paper Series are solely those of the author, and do not necessarily represent the views of the CIGS or its sponsor.

※ CIGS Working Paper Series is circulated in order to stimulate lively discussion and comments.

※Copyright belongs to the author(s) of each paper unless stated otherwise. 


\title{
Multiplicity of monetary steady states
}

\author{
Ryoji Hiraguchi*†and Keiichiro Kobayashi ${ }^{\ddagger}$
}

September 27, 2014

\begin{abstract}
In the Lagos-Wright model of money, monetary frictions alone cannot be a source of equilibrium multiplicity. However, the conclusion depends on the assumption that the agents always enter the centralized market after completing a transaction in the decentralized markets. In this paper, we investigate a monetary model in which the centralized market opens once, but the decentralized markets open twice in each period. We show that as the seller's money balances affect the buyer's problem in the first decentralized market, there may be multiple stationary equilibria.
\end{abstract}

Keywords: multiple equilibria; search; bargaining

JEL classification code: E5

${ }^{*}$ Faculty of Law and Economics, Chiba University. 1-33 Yayoi-cho, Inage-ku, Chiba, Japan. Email: ryojih@chiba-u.jp. Tel: 81-43-290-2399.

$\dagger$ The Canon Institute for Global Studies, Japan

${ }^{\ddagger}$ Faculty of Economics, Keio University. 2-15-45 Mita, Minato-ku, Tokyo, Japan. Email: kobayasi@econ.keio.ac.jp Tel: 81-3-5418-6703.

$\S$ The Canon Institute for Global Studies, Japan 


\section{Introduction}

The microfoundation of monetary models has been an important topic in macroeconomics. In a seminal paper by Lagos and Wright (2005), henceforth LW, a tractable monetary model is constructed in which the role of money is explicitly described. In LW, each period is divided into two subperiods, day and night. Money facilitates trades in the day market in which buyers and sellers are anonymous. The LW framework is now heavily used in monetary economics.

In LW, the uniqueness of the steady state is shown only under specific restrictions on the utility function. ${ }^{1}$ Recently, Wright (2010) relaxed the assumption and showed that the steady state is always unique. Wright (2010) also argued that monetary frictions alone cannot generate multiple equilibria.

In this note, we study a version of LW in which decentralized markets open twice in each period. Each date is divided into three subperiods - morning, afternoon, and night. The morning and afternoon markets are decentralized, while the night market is centralized. We show that for some utility functions, there are multiple stationary equilibria. This is because the objective function of the buyer in the morning market depends on the seller's money balances, and then the first-order conditions may not be monotone in the equilibrium money balances. The uniqueness result in Wright (2010) depends on the assumption that the agents always enter the centralized market after the transactions in the decentralized market. ${ }^{2}$

Our set-up is similar to that of Ennis (2009), who investigated a variant of LW by assuming that buyers may bypass the centralized market; however, he does not study equilibrium multiplicity.

In the following, Section 2 describes our model and Section3 shows the equilibrium multiplicity. Proofs are of the propositions are in the Appendix.

\footnotetext{
${ }^{1} \mathrm{LW}$ shows the uniqueness when the utility function in the day market $u$ satisfies $u^{\prime} u^{\prime \prime \prime} \leq\left(u^{\prime \prime}\right)^{2}$.

${ }^{2}$ Lagos and Wright (2003) studied a model which is similar to LW, but they showed that the steady states can be multiple. The difference between the two papers is that the former introduce real return on money, while the latter does not. Our model closely follows LW.
} 


\section{Model}

\section{$2.1 \quad$ Set-up}

Time is discrete and changes from $t=0$ to $+\infty$. There is a continuum of infinitely lived agents with unit measure. Each date is divided into three subperiods - morning, afternoon, and night. The morning and afternoon markets are decentralized and the night market is centralized. In the following, consecutive period variables are indexed by +1 .

At the beginning of each period, the agent receives an idiosyncratic preference shock. With probability $\alpha$, he becomes a Type-1 agent, who consumes in the morning market and produces in the afternoon market. Similarly, with probability $\alpha$, he becomes a Type-2 agent, who produces in the morning market and consumes in the afternoon market. The agent who consumes is called a buyer and the one who produces is called a seller. With probability $1-2 \alpha$, the agent becomes neither Type- 1 nor Type-2, and enters the night market directly. In Section 3.3, we investigate a case where the probability of becoming a buyer (and also a seller) in the morning market is independent of the one in the afternoon market.

In the morning market, the buyer obtains utility $u(q)$ from consuming $q$ units of output. The cost function of the seller is $q$. We assume that there exists $q^{*}>0$, such that $u^{\prime}\left(q^{*}\right)=1$. Similarly, in the afternoon market, the buyer obtains utility $\hat{u}(\hat{q})$ from consuming $\hat{q}$ units of output. The cost function of the seller is $\hat{q}$. We let $\hat{s}(q)=\hat{u}(q)-q$ denote the surplus. Let $\hat{q}^{*}>0$ be such that $\hat{u}^{\prime}\left(\hat{q}^{*}\right)=1$. In the night market, each agent obtains utility $U(c)$ from consuming $c$ units of general goods and disutility $h$ from producing $h$ units of goods. Let $c^{*}>0$ be such that $U^{\prime}\left(c^{*}\right)=1$. The function $u, \hat{u}$ and $U$ satisfy $u^{\prime}>0, \hat{u}^{\prime}>0, U^{\prime}>0, u^{\prime \prime}<0, \hat{u}^{\prime \prime}<0, U^{\prime \prime}<0$ and $u^{\prime}(0)=\hat{u}^{\prime}(0)=U^{\prime}(0)=+\infty$.

The role of money is to facilitate trades in the morning and afternoon markets, where buyers and sellers are anonymous. The buyers use money to pay. Money is divisible and storable, but it is intrinsically useless. The growth rate of the money supply $M$ is $\gamma$. 


\subsection{Night market}

We solve the model backward. At night, the agent solves

$$
W(m)=\max _{c, h}\left\{U(c)-h+\beta V_{+1}\left(m_{+1}\right)\right\}
$$

subject to the budget constraint $c=h+\phi\left(m+T-m_{+1}\right)$, where $\beta>0$ is a discount factor, $\phi$ is the price of money, $T=\gamma M$ is the lump-sum transfer, and $V(m)$ denotes the value function of the agents at the beginning of each period. The first-order conditions are $U^{\prime}(c)=1$ and

$$
\phi=\beta V_{+1}^{\prime}\left(m_{+1}\right) .
$$

As in LW, trades are efficient and the money balances at the beginning of each period are the same across agents. From the quasi-linearity of the utility function, $W$ is a linear function of the nominal balance, and $W(m)=\phi m+W(0)$.

\subsection{Afternoon market}

In the afternoon, the buyer (i.e., Type-2) makes a take-it-or-leave-it offer $(\hat{q}, \hat{d})$ to the seller (i.e., Type-1), where $\hat{q}$ is the quantity and $\hat{d}$ is the monetary transfer. The participation constraint of a seller is $W\left(m^{s}\right) \leq-\hat{q}+W\left(m^{s}+\hat{d}\right)$. As $W$ is linear, this is simplified as $\phi \hat{d}-\hat{q} \geq 0$. The buyer solves

$$
\hat{V}^{b}(m)=\max _{\hat{q}, \hat{d}}\{\hat{u}(\hat{q})+W(m-\hat{d})\} \quad \text { s.t } \hat{d} \leq m \text { and } \phi \hat{d}-\hat{q} \geq 0 .
$$

The buyer chooses the offer so that the participation constraint binds. Eq. (3) reduces to

$$
\hat{V}^{b}(m)=\max _{\hat{d}: \hat{d} \leq m}\{\hat{u}(\phi \hat{d})-\phi \hat{d}\}+\phi m+W(0)
$$

We let

$$
\hat{v}(x)=\hat{u}\left(\min \left\{x, \hat{q}^{*}\right\}\right)-\min \left\{x, \hat{q}^{*}\right\}+x .
$$

It satisfies $\hat{v}(x)=\hat{u}(x)$ if $x<\hat{q}^{*}$, and $\hat{v}(x)=\hat{s}\left(\hat{q}^{*}\right)+x$ if $x>\hat{q}^{*}$. Eq. (4) implies that $\hat{V}^{b}(m)=\hat{v}(\phi m)+W(0)$. Since the participation constraint is binding, the value function of the seller is $\hat{V}^{s}(m)=W(m)$. 


\begin{tabular}{|l|l|l|l|}
\hline & morning & afternoon & night \\
\hline Type-1 (buyer $\rightarrow$ seller $)$ & $m-d$ & $m-d+\hat{d}$ & $m_{+1}$ \\
\hline Type-2 (seller $\rightarrow$ buyer $)$ & $m+d$ & $m+d-\hat{d}$ & $m_{+1}$ \\
\hline
\end{tabular}

Table 1: Nominal balances

\subsection{Morning market}

In the morning, the buyer (i.e., Type-1) makes a take-it-or-leave-it offer $(q, d)$ to the seller (i.e., Type-2) where $(q, d)$ is the terms of trade. The participation constraint of a seller who has $m^{s}$ dollars is $\hat{V}^{b}\left(m^{s}+d\right)-q \geq \hat{V}^{b}\left(m^{s}\right)$. Table 1 summarizes the nominal balances of the agents at the end of each sub-period.

Let $V^{b}\left(m, m^{s}\right)$ denote the value function of the buyer who has $m$ dollars when the seller holds $m^{s}$ dollars. In the afternoon market, he becomes a seller with value function $W$. Since the participation constraint is binding, we obtain

$$
V^{b}\left(m, m^{s}\right)=\max _{d: d \leq m}\left\{u\left(\hat{V}^{b}\left(m^{s}+d\right)-\hat{V}^{b}\left(m^{s}\right)\right)-\phi d\right\}+\phi m+W(0) .
$$

As $u$ and $\hat{V}$ are increasing and concave, the objective function is concave in $d$. Therefore, the choice of $d$ is optimal if and only if it satisfies

$$
\hat{V}^{b \prime}\left(m^{s}+d\right) u^{\prime}\left(\hat{V}^{b}\left(m^{s}+d\right)-\hat{V}^{b}\left(m^{s}\right)\right) \geq \phi
$$

and the equality holds if $d<m$. The value function of the seller is $V^{s}(m)=\hat{V}^{b}(m)$.

As $V(m)=\alpha E_{m^{s}}\left[V^{b}\left(m, m^{s}\right)\right]+\alpha V^{s}(m)+(1-2 \alpha) W(m),(2)$ implies that

$$
i=\frac{\alpha}{\phi_{+1}}\left(E_{m^{s}}\left[\frac{\partial V_{+1}^{b}\left(m, m^{s}\right)}{\partial m}\right]+\frac{\partial V_{+1}^{s}(m)}{\partial m}\right)-2 \alpha,
$$

where $i=\frac{\phi}{\beta \phi_{+1}}-1$ is the nominal interest rate. In the steady state, $i=(1+\gamma) / \beta-1$. We let $z=\phi m$ be the real value of money balances. Similarly, we let $\rho=\phi d$ denote the real value of money transfer in the morning market. 


\section{Multiple equilibria}

Here we discuss steady states.

\subsection{Equilibrium condition}

First, we assume that the constraint $d \leq m$ binds in the morning. If $m^{s}=m$, from (6), $\frac{\partial V^{b}\left(m, m^{s}\right)}{\partial m}=\hat{V}^{b \prime}(2 m) u^{\prime}\left(\hat{V}^{b}(2 m)-\hat{V}^{b}(m)\right)$. As $\hat{V}^{b \prime}(m)=V^{s \prime}(m)=\phi \hat{v}^{\prime}(\phi m)$, (8) reduces to

$$
i=\alpha\left\{\hat{v}^{\prime}(2 z) u^{\prime}(\hat{v}(2 z)-\hat{v}(z))+\hat{v}^{\prime}(z)-2\right\}
$$

The constraint $d \leq m$ is binding if and only if $\hat{V}^{b \prime}(2 m) u^{\prime}\left(\hat{V}^{b}(2 m)-\hat{V}^{b}(m)\right) \geq \phi$. This is simplified as $\hat{v}^{\prime}(2 z) u^{\prime}(\hat{v}(2 z)-\hat{v}(z)) \geq 1$.

Next we assume $d<m$. From the envelope theorem, $\frac{\partial V^{b}}{\partial m}=1$. Eq. (8) reduces to

$$
i=\alpha\left\{1+\hat{v}^{\prime}(z)-2\right\}
$$

Since $d<m, \rho<z$. Further, Eq. (7) is written as $\hat{v}^{\prime}(z+\rho) u^{\prime}(\hat{v}(z+\rho)-\hat{v}(\rho))=1$. As $u^{\prime}(0)=+\infty$, there exists such $\rho$, if and only if $\hat{v}^{\prime}(2 z) u^{\prime}(\hat{v}(2 z)-\hat{v}(z))<1$.

A stationary equilibrium $(\phi, q, \hat{q}, d, \hat{d})$ is a tuple in which $\phi$ is the price of money, $q(\hat{q})$ is the quantity in the morning market (afternoon market), and $d(\hat{d})$ is the transfer in the morning market (afternoon market).

We obtain the following results.

Proposition 1 A stationary equilibrium $(\phi, q, \hat{q}, d, \hat{d})$ exists if the following conditions are satisfied:

1) the real balance $z=\phi M$ satisfies $i=\alpha\left[\max \left\{\hat{v}^{\prime}(2 z) u^{\prime}(\hat{v}(2 z)-\hat{v}(z)), 1\right\}+\hat{v}^{\prime}(z)-2\right]$, where $i=(1+\gamma) / \beta-1$, and $\gamma$ is the money growth rate.

2) the quantities $q$ and $\hat{q}$ and the money transfers $d$ and $\hat{d}$ satisfy the following condition:

a) if $\hat{v}^{\prime}(2 z) u^{\prime}(\hat{v}(2 z)-\hat{v}(z)) \geq 1, q=\hat{v}(2 z)-\hat{v}(z), \phi d=z$ and $\hat{q}=\phi \hat{d}=\min \left\{2 z, \hat{q}^{*}\right\}$.

b) if $\hat{v}^{\prime}(2 z) u^{\prime}(\hat{v}(2 z)-\hat{v}(z))<1, q=\hat{v}(z+\rho)-\hat{v}(z), \phi d=\rho$ and $\hat{q}=\phi \hat{d}=\min \left\{z+\rho, \hat{q}^{*}\right\}$, where $\rho$ is such that $\rho \leq z$ and $\hat{v}^{\prime}(z+\rho) u^{\prime}(\hat{v}(z+\rho)-\hat{v}(z))=1$.

3) the price of money evolves according to $\phi / \phi_{+1}=1+\gamma$. 
The stationary welfare $W=u(q)-q+\hat{u}(\hat{q})-\hat{q}$ is maximized if $q=q^{*}$ and $\hat{q}=\hat{q}^{*}$. The next proposition deals with the optimal policy.

Proposition 2 The Friedman rule is the optimal monetary policy.

\subsection{Number of equilibria}

Let $g(x)=\max \left\{\hat{v}^{\prime}(2 x) u^{\prime}(\hat{v}(2 x)-\hat{v}(x)), 1\right\}+\hat{v}^{\prime}(x)$. Then, $z$ solves $\alpha(g(z)-2)=i$. or equivalently $g(z)=i / \alpha+2$. We focus on the CRRA functions $u(q)=A \frac{q^{1-\sigma}}{1-\sigma}$ and $\hat{u}(\hat{q})=\frac{\hat{q}^{1-\theta}}{1-\theta}$, with $\sigma=\theta=2$. In this case, $\hat{q}^{*}=1$; if $x<1, \hat{v}(x)=-1 / x$, and if $x \geq 1$, $\hat{v}(x)=x-2$. Thus,

1) If $0<z<1 / 2$, then $g(z)=\max \{A, 1\}+\frac{1}{z^{2}}$.

2) If $1 / 2 \leq z \leq 1$, then $g(z)=\max \left\{A\left(\frac{1}{z}+2 z-2\right)^{-2}, 1\right\}+\frac{1}{z^{2}}$.

3) If $z>1$, then $g(z)=\max \left\{\frac{A}{z^{2}}, 1\right\}+1$.

The function $g$ may be non-monotone if $z \in[1 / 2,1]$, because the function $1 / z+2 z$ is U-shaped.

Proposition 3 Suppose $u(q)=A \frac{q^{1-\sigma}}{1-\sigma}$ and $\hat{u}(x)=\frac{x^{1-\theta}}{1-\theta}$. If $\sigma=\theta=2$ and $A>4$, there are three steady state equilibria for some $i$.

Figure 1 illustrates the case where $A=9$ and $\alpha=0.5 \%$. It shows that if $i=6 \%$, there are three equilibria, $E_{1}, E_{2}$, and $E_{3}$.

The equilibrium can be multiple because the buyer's objective function in (6) depends on and may be non-concave in the seller's nominal balance $m^{s}$. In LW, the centralized market always follows the decentralized market, and the seller's nominal balance is independent of the buyer's objective function due to the quasi-linearity of the utility function. Thus, the equilibrium is unique in LW.

\subsection{Extension}

Thus far, we assumed that the role of the agent in the afternoon is determined at the beginning of the morning. Here, we assume that the preference shocks are independent. In 


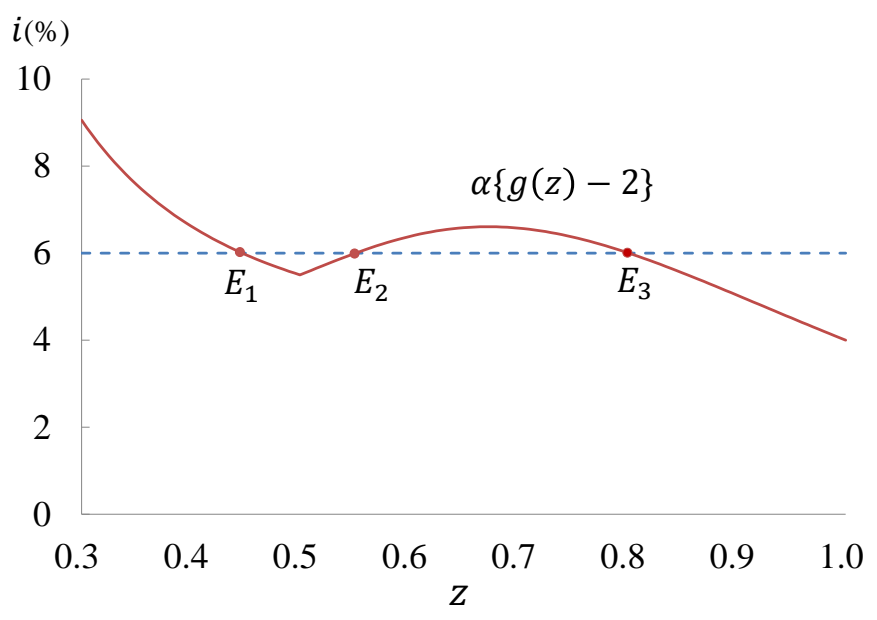

Figure 1: Multiple equilibria

the morning market, with probability $\alpha$, the agent becomes a buyer and with probability $\alpha$, he becomes a seller. With probability $1-2 \alpha$, the agent becomes neither buyer nor seller. In the afternoon market, the agent becomes a buyer with probability $\hat{\alpha}$ and a seller with probability $\hat{\alpha}$. With probability $1-2 \hat{\alpha}$, the agent enters the night market directly. If $\hat{\alpha}=0$, the model coincides with the one in LW. As in the previous section, we assume that the utility function in the morning market is $u(q)=A \frac{q^{1-\sigma}}{1-\sigma}$ and the one in the afternoon market is $\hat{u}(x)=\frac{x^{1-\theta}}{1-\theta}$.

Proposition 4 When the preference shocks are independent, there are two stationary equilibria for some parameter values.

In this set-up, every agent has a positive probability of becoming a buyer at the beginning of each period. In this case, from the Inada condition $\hat{u}^{\prime}(0)=\infty$, the buyer's constraint $d \leq m$ in the morning market is never binding. This differs from the previous model. 


\section{Appendix}

In the Appendix, we provide proofs for the propositions.

\section{A Proof for Proposition 1}

Eqs. (9) and (10) imply $i=\alpha\left[\max \left\{\hat{v}^{\prime}(2 z) u^{\prime}(\hat{v}(2 z)-\hat{v}(z)), 1\right\}+\hat{v}^{\prime}(z)-2\right]$. If $\hat{v}^{\prime}(2 z) u^{\prime}(\hat{v}(2 z)-$ $\hat{v}(z)) \geq 1$, then $\rho=z$. In the afternoon, the real balances of the buyer are $2 z$. The quantities are $q=\hat{v}(2 z)-\hat{v}(z)$ and $\hat{q}=\min \left\{2 z, q^{*}\right\}$. If $\hat{v}^{\prime}(2 z) u^{\prime}(\hat{v}(2 z)-\hat{v}(z))<1$, then $\rho$ satisfies the first-order condition. In the afternoon the real balances of the buyer are $z+\rho$. Thus, $q=\hat{v}(z+\rho)-\hat{v}(z)$ and $\hat{q}=\min \left\{z+\rho, q^{*}\right\}$.

\section{B Proof for Proposition 2}

As $\hat{v}^{\prime}(z) \geq 1$ for all $z$ and $\hat{v}^{\prime}(z)=1$ only if $z \geq \hat{q}^{*}, i=0$ if and only if $z \geq \hat{q}^{*}$ and $\hat{v}^{\prime}(2 z) u^{\prime}(\hat{v}(2 z)-\hat{v}(z)) \leq 1$. If $z \geq \hat{q}^{*}$, then $\hat{q}=\hat{q}^{*}$. Moreover, $\hat{v}(2 z)=\hat{s}\left(\hat{q}^{*}\right)+2 z$, $\hat{v}(z)=\hat{s}\left(\hat{q}^{*}\right)+z$ and $\hat{v}^{\prime}(z)=\hat{v}^{\prime}(z+\rho)=1$. Thus, $\hat{v}^{\prime}(z+\rho) u^{\prime}(\hat{v}(z+\rho)-\hat{v}(z))=u^{\prime}(\rho)=1$ implies $\rho=q^{*}$ and then $q=q^{*}$. If we take $z \geq q^{*}$, then we can take such $\rho$. This implies that if we take a sufficiently large $z, q=q^{*}$ and $\hat{q}=\hat{q}^{*}$.

\section{Proof for Proposition 3}

If $z<0.5, g(z)=A+\frac{1}{z^{2}}$ is decreasing in $z$. If $0.5 \leq z<1, \hat{v}(2 z)-\hat{v}(z)=-2+\frac{1}{z}+2 z$. As $A>4, A(-2+1 / z+2 z)^{-2}>1$, and then $g(z)=A(-2+1 / z+2 z)^{-2}+\frac{1}{z^{2}}$. We have $g^{\prime}(z)=2 A\left(z^{-2}-2\right)(1 / z+2 z-2)^{-3}-2 z^{-3}$ and $g^{\prime}(1 / 2)=4 A-16>0$. Thus, $\hat{v}(z)$ is strictly increasing in $z$ if $z$ is sufficiently close to $1 / 2$. Note that $g(1 / 2)>2$. If $z>1 / \sqrt{2}$, then $g^{\prime}(z)<0$. If $z>1$, then $g(z)=\max \left\{u^{\prime}(z), 1\right\}+1=\max \left\{A z^{-2}, 1\right\}+1$. This is a decreasing function if $z<\sqrt{A}$, and $g(z)=2$ if $z>\sqrt{A}$. Therefore, if $2(1+i)=g(1 / 2)+\epsilon$, for sufficiently small $\epsilon>0$, there are three values of $z$ that solve $g(z)=2(i+1)$. 


\section{Proof for Proposition 4}

The structure of the night market is the same as before. The value function of the agent at night is $w(m)=\phi m+w(0)$, and the value function of the agent at the beginning of the afternoon market is $\hat{F}(m)=\hat{\alpha} \hat{F}^{b}(m)+(1-\hat{\alpha}) w(m)$, where $\hat{F}^{b}(m)$ is the value function of the buyer in the afternoon market. This simplifies to $\hat{F}(m)=\hat{f}(\phi m)+w(0)$, where $\hat{f}(z)=\hat{\alpha}\left[\hat{u}\left(\min \left\{z, \hat{q}^{*}\right\}\right)-\min \left\{z, \hat{q}^{*}\right\}\right]+z$.

In the morning market, the buyer makes a take-it-or-leave-it offer $(q, d)$ to the seller. The participation constraint is $\hat{F}\left(m^{s}+d\right)-\hat{F}\left(m^{s}\right) \geq q$. The buyer solves

$$
F^{b}\left(m, m^{s}\right)=\max _{d: d \leq m}\left\{u\left(\hat{F}^{b}\left(m^{s}+d\right)-\hat{F}\left(m^{s}\right)\right)+\hat{F}(m-d)\right\}
$$

The level of $d$ is optimal if it satisfies the first-order condition:

$$
\hat{F}^{\prime}\left(m^{s}+d\right) u^{\prime}\left(\hat{F}\left(m^{s}+d\right)-\hat{F}\left(m^{s}\right)\right)=\hat{F}^{\prime}(m-d) .
$$

From the Inada condition $u^{\prime}(0)=\hat{u}^{\prime}(0)=\hat{F}^{\prime}(0)=+\infty$, the solution exists and is interior. Thus, the constraint $d \leq m$ never binds. The seller's value function is $F^{s}(m)=\hat{F}(m)$.

The value function in the morning is $F(m)=\alpha V^{b}\left(m, m^{s}\right)+(1-\alpha) \hat{F}(m)$. We use the envelop theorem to obtain $F^{\prime}(m)=\alpha \hat{F}^{\prime}(m-d)+(1-\alpha) \hat{F}^{\prime}(m)$. In the steady state, the first-order conditions $\phi=\beta F_{+1}^{\prime}\left(m_{+1}\right)$ and (12) reduce to

$$
\begin{aligned}
1+i & =\alpha \hat{f}^{\prime}(z-\rho)+(1-\alpha) \hat{f}^{\prime}(z), \\
\hat{f}^{\prime}(z-\rho) & =\hat{f}^{\prime}(z+\rho) u^{\prime}(\hat{f}(z+\rho)-\hat{f}(z)) .
\end{aligned}
$$

Eqs. (13) and (14) determine $z$ and $\rho$.

Transactions in the afternoon depend on the buyer's role in the morning. If he was also a buyer, his real balances at the beginning of the afternoon market, say $\phi \hat{m}$, are equal to $z+\rho$ and the quantity is $\hat{q}=\hat{q}_{1} \equiv \min \left\{z+\rho, \hat{q}^{*}\right\}$. If he was a seller, $\phi \hat{m}=z-\rho$ and $\hat{q}=\hat{q}_{2} \equiv \min \left\{z-\rho, \hat{q}^{*}\right\}$. If he was neither a buyer nor a seller, $\phi \hat{m}=z$ and $\hat{q}=\hat{q}_{3} \equiv \min \left\{z, \hat{q}^{*}\right\}$. The real money transfer $\phi \hat{d}$ is always equal to the quantity. $A$ stationary equilibrium is a vector $\left(\phi, d, q, \hat{q}_{1}, \hat{q}_{2}, \hat{q}_{3}\right)$, such that the real balance $z=\phi M$ 
and the real money transfer in the morning market $\rho=\phi d$ satisfy (13) and (14), and $q=\hat{f}(z+\rho)-\hat{f}(z), \hat{q}_{1}=\min \left\{z+\rho, \hat{q}^{*}\right\}, \hat{q}_{2}=\min \left\{z-\rho, \hat{q}^{*}\right\}$ and $\hat{q}_{3}=\min \left\{z, \hat{q}^{*}\right\}$.

We assume that $u(q)=A \frac{q^{1-\sigma}}{1-\sigma}$ and $\hat{u}(\hat{q})=\frac{\hat{q}^{1-\theta}}{1-\theta}$. We focus on the equilibria where the real balances of all the buyers in the afternoon market are below $\hat{q}^{*}$. This happens if $\rho+z<1$. We obtain $\hat{f}(y)=\hat{\alpha} \frac{y^{1-\theta}}{1-\theta}+(1-\hat{\alpha}) y$ if $y=z-\rho, z$ and $z+\rho$. If we set $\alpha=\hat{\alpha}=0.5, \theta=2$ and let $x=\frac{\rho}{z} \in(0,1)$, then (13) and (14) are simplified as

$$
\begin{aligned}
z & =z(x) \equiv b \sqrt{(1-x)^{-2}+1}, \\
\frac{(1-x)^{-2}+z^{2}}{(1+x)^{-2}+z^{2}} & =A\left(\frac{x}{z(1+x)}+z x\right)^{-\sigma} .
\end{aligned}
$$

with $b=1 / \sqrt{2(1+2 i)}$. Substituting (15) into (16) yields

$$
\omega(x) \equiv \ln \left[\frac{x(1-x)}{h(x)(1+x)}+b^{2} \frac{x h(x)}{1-x}\right]+\frac{1}{\sigma} \ln \left[\frac{(1-x)^{-2}+z(x)^{2}}{(1+x)^{-2}+z(x)^{2}}\right]=\ln b+\frac{\ln A}{\sigma},
$$

where $h(x)=\sqrt{(1-x)^{2}+1}$. We show that (17) can have two solutions that satisfy

$$
\rho+z=(1+x) z(x)<1 \text {. }
$$

We let $\omega_{1}(x)=\ln \left[\frac{x(1-x)}{h(1+x)}+b^{2} \frac{x h}{1-x}\right]$ denote the first term of $\omega$. If $b=0, \omega_{1}$ is inverted-U shaped, and $\omega_{1}(x)=C_{1}$ has two solutions for some $C_{1}$. If $b=0,(18)$ obviously holds. Thus, if $b$ is sufficiently small, there are two solutions to $\omega_{1}(x)=C_{2}$ for some $C_{2}$, and each satisfies (18). Fix such $b$ and $C_{2}$. If $\sigma=+\infty, \omega(x)=\omega_{1}(x)$. Thus, if we take a sufficiently large $\sigma$ and let $\ln A=\sigma\left(C_{2}-\ln b\right), \omega(x)=\ln b+\frac{\ln A}{\sigma}$ has two solutions and each satisfies (18). One can easily check that there are two solutions when $b=0.026$, $\sigma=3$ and $\ln A=18.8$.

\section{References}

Ennis, H., 2009. Avoiding the inflation tax. International Economic Review 50, 607-625. Lagos, R., Wright, R., 2005. A unified framework for monetary theory and policy analysis. Journal of Political Economy 113, 463-484.

R. Wright., 2010. A uniqueness proof for monetary steady state. Journal of Economic Theory 145, 382-391. 